\title{
REMARKS ON THE UNIVALENCE CRITERION OF PASCU AND PASCU
}

\author{
VAIDHYANATHAN BHARANEDHAR and SAMINATHAN PONNUSAMY ${ }^{凶}$
}

(Received 27 January 2013; accepted 11 May 2013; first published online 22 August 2013)

\begin{abstract}
We consider a recent work of Pascu and Pascu ['Neighbourhoods of univalent functions', Bull. Aust. Math. Soc. 83(2) (2011), 210-219] and rectify an error that appears in their work. In addition, we study certain analogous results for sense-preserving harmonic mappings in the unit disc $|z|<1$. As a corollary to this result, we derive a coefficient condition for a sense-preserving harmonic mapping to be univalent in $|z|<1$.
\end{abstract}

2010 Mathematics subject classification: primary 30C45; secondary 30C50, 30C55.

Keywords and phrases: analytic, univalent, convex, close-to-convex and harmonic mappings.

\section{Introduction and preliminaries}

The well-known Noshiro-Warschawski-Wolff criterion (see [3, page 47]) for univalency asserts the following.

Theorem A. If $f: D \rightarrow \mathbb{C}$ is analytic in a convex domain $D$ and $\operatorname{Re} f^{\prime}(z)>0$ for all $z \in D$, then $f$ is univalent in $D$.

As a counterpart of this result Pascu and Pascu [6] proved the following lemma.

Lemma B [6, Proposition 2.1]. Let $f: D \rightarrow \mathbb{C}$ be an analytic function in the domain $D$ and define

$$
K(f, D)=\inf _{\substack{a \neq b \\ a, b \in D}}\left|\frac{f(a)-f(b)}{a-b}\right| .
$$

(1) If $K(f, D)>0$, then $f$ is univalent in $D$.

(2) Conversely, if $f$ is univalent in $D$ and $\Omega \subset \bar{\Omega} \subset D$ is a domain strictly contained in $D$, then $K(f, \Omega)>0$.

The research of V. Bharanedhar was supported by a fellowship of the University Grants Commission, India. The second author is currently at the Indian Statistical Institute (ISI), Chennai Centre, SETS (Society for Electronic Transactions and Security), MGR Knowledge City, CIT Campus, Taramani, Chennai 600 113, India.

(C) 2013 Australian Mathematical Publishing Association Inc. 0004-9727/2013 \$16.00 
It is worth pointing out that the converse result, namely item (2) in Lemma B, is not necessarily true. For example, consider $f(z)=e^{z}$ in the strip $D=\{z:-\pi<\operatorname{Im} z<\pi\}$. It is a simple exercise to see that $f$ is univalent in $D$. Also let $\Omega=\{z:-\pi / 2<\operatorname{Im} z<\pi / 2\}$ so that $\Omega \subset \bar{\Omega} \subset D$ and $\{-n: n \in \mathbb{N}\} \subset \Omega$. Moreover, since the sequence $\left\{e^{-n}\right\}$ converges to 0 , given $\epsilon>0$ we can find a stage $N \in \mathbb{N}$ such that

$$
\left|\frac{e^{-n}-e^{-m}}{n-m}\right| \leq\left|e^{-n}-e^{-m}\right|<\epsilon \text { for all } n, m \geq N .
$$

This observation shows that

$$
K(f, \Omega)=\inf _{\substack{a \neq b \\ a, b \in \Omega}}\left|\frac{e^{-a}-e^{-b}}{a-b}\right|=0,
$$

from which we obtain that the converse part of Lemma B fails. The main mistake in the proof of part (2) of Lemma B comes from the fact that Pascu and Pascu implicitly assumed in their argument that the domain $D$ is bounded. If this were made an explicit condition then their result would be correct.

In addition, the authors in [6] proved the following result.

Theorem C [6, Theorem 2.4]. Let $f: D \rightarrow \mathbb{C}$ be a nonconstant analytic function in the convex domain $D$. If there exists an analytic function $g: D \rightarrow \mathbb{C}$ univalent in $D$ such that

$$
\left|f^{\prime}(z)-g^{\prime}(z)\right| \leq K(g, D), \quad z \in D,
$$

then the function $f$ is also univalent in $D$.

As a consequence of Theorem $\mathrm{C}$, they obtained the following corollary.

Corollary D [6, Corollary 2.6]. If $f: D \rightarrow \mathbb{C}$ is nonconstant and analytic in the convex domain $D$ and there exists $c>0$ such that

$$
\left|f^{\prime}(z)-c\right| \leq c, \quad z \in D
$$

then $f$ is univalent in $D$.

Moreover, Pascu and Pascu remarked [6, Remark 2.7] that Corollary D is equivalent to Theorem A. It can easily be seen that Theorem A implies Corollary D, but again the converse is not necessarily true as the next example demonstrates.

Example 1.1. Let $D$ be the right half-plane $\{z \in \mathbb{C}: \operatorname{Re} z>0\}$ and consider the function $f(z)=z^{2}$. Then $f^{\prime}(z)=2 z$ and $\operatorname{Re} f^{\prime}(z)>0$ in $D$. Clearly, by the NoshiroWarschawski-Wolff univalence criterion $f$ is univalent in $D$. On the other hand, univalency of $f$ in $D$ does not follow from Corollary $\mathrm{D}$, because we cannot find a universal constant $c>0$ satisfying (1.1). Thus the observation made by the authors in [6] about the converse of Corollary D is not true in general.

In Section 2, we extend Theorem $\mathrm{C}$ for sense-preserving harmonic univalent mappings and present a number of corollaries, remarks and examples. 


\section{Main results}

A complex-valued function $f=u+i v$ in a simply connected domain $D$ is said to be harmonic if the real and imaginary parts of $f$ satisfy Laplace's equation. In $D, f$ has the canonical decomposition $f=h+\bar{g}$, where $h$ and $g$ are analytic in $D$. The Jacobian $J_{f}$ of $f$ is given by

$$
J_{f}(z)=\left|h^{\prime}(z)\right|^{2}-\left|g^{\prime}(z)\right|^{2} .
$$

We say that $f$ is sense-preserving in $D$ if $J_{f}(z)>0$, for all $z \in D$. If the Jacobian of $f$ is nonvanishing in $D$, then by the inverse mapping theorem it follows that $f$ is locally univalent in $\mathbb{D}$. For harmonic functions the converse is also true as asserted by Lewy's theorem [5] (see also [4, page 20]). We refer to Clunie and Sheil-Small [2] and Duren [4] for many important results on harmonic univalent mappings.

In [7], the authors considered the class

$$
C_{H}^{1}:=\left\{f=h+\bar{g}, f(0)=f_{z}(0)=1 \text { and } f_{\bar{z}}(0)=0: \operatorname{Re} h^{\prime}(z)>\left|g^{\prime}(z)\right|, z \in \mathbb{D}\right\},
$$

where $\mathbb{D}=\{z:|z|<1\}$ is the open unit disc in $\mathbb{C}$. They proved that the functions in $C_{H}^{1}$ are not only univalent in $\mathbb{D}$ but also close-to-convex in $\mathbb{D}$ (see [7, Lemma 1.1]). This result is regarded as a harmonic analogue of the Noshiro-Warschawski-Wolff criterion.

THEOREM 2.1. Let $f: D \rightarrow \mathbb{C}$ be a sense-preserving harmonic function in a convex domain $D$ with the canonical decomposition $f=h+\bar{g}$. If there exists an analytic univalent function $\phi: D \rightarrow \mathbb{C}$ such that

$$
\left|h^{\prime}(z)-\phi^{\prime}(z)\right|+\left|g^{\prime}(z)\right| \leq K(\phi, D), \quad z \in D,
$$

then $f$ is univalent in $D$.

Proof. Assume that $f$ is not univalent in $D$. Then there are points $z_{1}, z_{2} \in D$ such that $z_{1} \neq z_{2}$ and $f\left(z_{1}\right)=f\left(z_{2}\right)$. Since $D$ is convex, the line segment joining $z_{1}$ and $z_{2}$ lies completely in $D$, that is, $\left\{z(t)=(1-t) z_{1}+t z_{2}: 0 \leq t \leq 1\right\} \subset D$. An integration along this line segment, together with (2.1), yields

$$
\begin{aligned}
\left|\phi\left(z_{2}\right)-\phi\left(z_{1}\right)\right| & =\left|\left(f\left(z_{2}\right)-\phi\left(z_{2}\right)\right)-\left(f\left(z_{1}\right)-\phi\left(z_{1}\right)\right)\right| \\
& =\left|\int_{0}^{1} \frac{d}{d t}(f(z(t))-\phi(z(t))) d t\right| \\
& =\left|\int_{0}^{1}\left(\left(h^{\prime}(z(t))-\phi^{\prime}(z(t))\right)\left(z_{2}-z_{1}\right)+\overline{g^{\prime}(z(t))\left(z_{2}-z_{1}\right)}\right) d t\right| \\
& \leq \int_{0}^{1}\left(\left|h^{\prime}(z(t))-\phi^{\prime}(z(t))\right|+\left|g^{\prime}(z(t))\right|\right)\left|z_{2}-z_{1}\right| d t \\
& \leq K(\phi, D)\left|z_{2}-z_{1}\right| .
\end{aligned}
$$


Since $z_{1} \neq z_{2}$, from the above inequality and the definition of $K(\phi, D)$, as in [6],

$$
K(\phi, D)=\left|\frac{\phi\left(z_{2}\right)-\phi\left(z_{1}\right)}{z_{2}-z_{1}}\right| .
$$

Again following the method of proof of [6], we consider the auxiliary function $P$ defined on $D \backslash\left\{z_{2}\right\}$ by

$$
P(z)=\frac{\phi(z)-\phi\left(z_{2}\right)}{z-z_{2}}, \quad z \in D \backslash\left\{z_{2}\right\} .
$$

As $\phi$ is analytic in $D$, it follows that $P$ is analytic in $D \backslash\left\{z_{2}\right\}$ and we see that the limit

$$
\lim _{z \rightarrow z_{2}} P(z)=\lim _{z \rightarrow z_{2}} \frac{\phi(z)-\phi\left(z_{2}\right)}{z-z_{2}}=\phi^{\prime}\left(z_{2}\right)
$$

exists and is finite. Therefore, we can extend the function $P$ to an analytic function in $D$, which we also denote by $P$. Since

$$
\inf _{z \in D}|P(z)|=\inf _{\substack{z \neq z_{2} \\ z \in D}}|P(z)|=\inf _{\substack{z \neq z_{2} \\ z \in D}}\left|\frac{\phi(z)-\phi\left(z_{2}\right)}{z-z_{2}}\right| \geq \inf _{\substack{a \neq b \\ a, b \in D}}\left|\frac{\phi(a)-\phi(b)}{a-b}\right|=K(\phi, D),
$$

it follows from (2.2) that

$$
\inf _{z \in D}|P(z)| \geq K(\phi, D)=\left|\frac{\phi\left(z_{2}\right)-\phi\left(z_{1}\right)}{z_{2}-z_{1}}\right|=\left|P\left(z_{1}\right)\right| \geq \inf _{z \in D}|P(z)| .
$$

Thus, the minimum modulus value of $P$ in $D$ is attained at $z_{1}$.

Since $\phi$ is univalent in $D$, it follows that $P$ is a nonvanishing analytic function in $D$ which attains its minimum modulus value in the interior of $D$. Hence, by the minimum modulus principle for nonvanishing analytic functions, it follows that $P$ must be constant in $D$.

Thus,

$$
\phi(z)=c\left(z-z_{2}\right)+\phi\left(z_{2}\right), \quad z \in D,
$$

for a certain constant $c \in \mathbb{C}$. From the definition of $P$, one can easily see that $c=\phi^{\prime}\left(z_{2}\right) e^{i \theta}$ for some $\theta \in \mathbb{R}$. From (2.3) we see that $\phi$ is a linear function and so a simple computation shows that $K(\phi, D)=|c|$ in this case.

As a consequence of the above discussion, (2.1) becomes

$$
\left|h^{\prime}(z)-c+\overline{g^{\prime}(z)}\right| \leq\left|h^{\prime}(z)-c\right|+\left|g^{\prime}(z)\right| \leq|c|, \quad z \in D .
$$

We need to deal with two cases.

Case (i). Suppose that equality holds in both the inequalities in (2.4) for a particular point, say at $z_{0} \in D$. Now, by the maximum modulus principle for complex-valued harmonic functions (see [1, Corollary 1.11, page 8]),

$$
h^{\prime}(z)=l-\overline{g^{\prime}(z)}, \quad z \in D,
$$

where $l \in \mathbb{C}$. Since $h^{\prime}$ is an analytic function, it follows that $g^{\prime}$ is constant and so is $h^{\prime}$. Further, from the sense-preserving property of $f$, we get $f(z)=\alpha z+\beta \bar{z}+\gamma$ for some $\alpha, \beta$ and $\gamma \in \mathbb{C}$ with $|\alpha|>|\beta|$. 
Case (ii). Suppose Case (i) does not happen. Now, repeating the above proof with $\phi(z)=c z$,

$$
\begin{aligned}
\left|c z_{2}-c z_{1}\right| & =\left|\left(f\left(z_{2}\right)-c z_{2}\right)-\left(f\left(z_{1}\right)-c z_{1}\right)\right| \\
& =\left|\int_{0}^{1} \frac{d}{d t}(f(z(t))-c z(t)) d t\right| \\
& =\left|\int_{0}^{1}\left(\left(h^{\prime}(z(t))-c\right)\left(z_{2}-z_{1}\right)+\overline{e^{i \theta} g^{\prime}(z(t))}\left(z_{2}-z_{1}\right)\right) d t\right| \text { for some } \theta \in \mathbb{R}, \\
& \leq \int_{0}^{1}\left|h^{\prime}(z(t))-c+\overline{e^{i \theta} g^{\prime}(z(t))}\right|\left|z_{2}-z_{1}\right| d t \\
& <|c|\left|z_{2}-z_{1}\right|,
\end{aligned}
$$

which is a contradiction, where in the above $\theta=2 \arg \left(z_{2}-z_{1}\right)$. Indeed, if we have equality in the last inequality, then as in Case (i) it is easy to see that $f$ is an affine mapping. This contradiction shows that the function $f$ is univalent in $D$.

Remark 2.2. The sense-preserving assumption about $f$ cannot be removed in Theorem 2.1. For example, consider the harmonic function $f(z)=\operatorname{Re} z, z \in \mathbb{D}$. The Jacobian of $f$ is zero on $\mathbb{D}$, which shows that $f$ is not even sense-preserving. Now take $\phi(z)=z / 2$; then $(2.1)$ is satisfied with $K(\phi, \mathbb{D})=1 / 2$ but $f$ is not univalent in $\mathbb{D}$.

Remark 2.3. The right-hand side in (2.1) cannot be replaced by a larger quantity, as can be seen by the function $f(z)=z+a \bar{z}^{2}$ in the unit disc $\mathbb{D}$, where $a \in \mathbb{D}$. For if we take $\phi(z)=z$, then $K(\phi, \mathbb{D})=1$ and hence, using Theorem 2.1, we get that $f$ is univalent in $\mathbb{D}$ if $|2 a z| \leq 1$ for all $z \in \mathbb{D}$, that is, if $|2 a| \leq 1$. But using a direct computation, one can see that $f$ is univalent in $\mathbb{D}$ if and only if $|2 a| \leq 1$. Hence inequality (2.1) in Theorem 2.1 is sharp. Here we note that if $|2 a| \leq 1$ then $f \in C_{H}^{1}$ and hence $f$ is close-to-convex on $\mathbb{D}$.

Corollary 2.4. Let $f: D \rightarrow \mathbb{C}$ be a sense-preserving harmonic function in a convex domain $D$ with the canonical decomposition $f=h+\bar{g}$. If there exists a constant $c>0$ such that

$$
\left|h^{\prime}(z)-c\right|+\left|g^{\prime}(z)\right| \leq c, \quad z \in D,
$$

then $f$ is univalent in $D$.

Proof. The proof follows from Theorem 2.1 by taking $\phi(z)=c z$ with $c>0$.

Corollary 2.5. Let $\phi: \mathbb{D} \rightarrow \mathbb{C}$ be an analytic univalent function with Taylor series expansion

$$
\phi(z)=\sum_{n=0}^{\infty} k_{n} z^{n}, \quad z \in \mathbb{D} .
$$

Let $f$ be a sense-preserving harmonic mapping with the canonical decomposition

$$
f(z)=\sum_{n=1}^{\infty} a_{n} z^{n}+\sum_{n=1}^{\infty} \overline{b_{n}} \bar{z}^{n}, \quad z \in \mathbb{D} .
$$


If the coefficients in (2.5) satisfy

$$
\sum_{n=1}^{\infty} n\left|a_{n}-k_{n}\right|+\sum_{n=1}^{\infty} n\left|b_{n}\right| \leq K(\phi, \mathbb{D})
$$

then $f$ is univalent in $\mathbb{D}$.

PROOF. Let $h(z)=\sum_{n=1}^{\infty} a_{n} z^{n}$ and $g(z)=\sum_{n=1}^{\infty} b_{n} z^{n}$. Then $f=h+\bar{g}$. Now

$$
\begin{aligned}
\left|h^{\prime}(z)-\phi^{\prime}(z)\right|+\left|g^{\prime}(z)\right| & =\left|\sum_{n=1}^{\infty} n a_{n} z^{n-1}-\sum_{n=1}^{\infty} n k_{n} z^{n-1}\right|+\left|\sum_{n=1}^{\infty} n b_{n} z^{n-1}\right| \\
& \leq \sum_{n=1}^{\infty} n\left|a_{n}-k_{n}\right||z|^{n-1}+\sum_{n=1}^{\infty} n\left|b_{n}\right||z|^{n-1} \\
& <\sum_{n=1}^{\infty} n\left|a_{n}-k_{n}\right|+\sum_{n=1}^{\infty} n\left|b_{n}\right| \\
& \leq K(\phi, \mathbb{D}),
\end{aligned}
$$

for all $z \in \mathbb{D}$. Thus, by Theorem 2.1 , we conclude that $f$ is univalent in $\mathbb{D}$.

ExAmple 2.6. If we take $\phi(z)=z$ in Corollary 2.5, then it follows easily that the harmonic function $f(z)=z+a \bar{z}^{n} \quad(n \geq 2)$ is univalent in $\mathbb{D}$ whenever $|a| \leq 1 / n$ (as pointed out in Remark 2.3).

ExAmple 2.7. Let $\alpha$ be such that $\alpha \in(0,1)$ and consider the function

$$
\varphi(z)=\frac{z-\alpha}{1-\alpha z}, \quad z \in \mathbb{D} .
$$

It is well known that $\varphi$ is an analytic automorphism of the unit disc and

$$
K(\varphi, \mathbb{D})=\inf _{\substack{a \neq b \\ a, b \in \mathbb{D}}}\left|\frac{\varphi(a)-\varphi(b)}{a-b}\right|=\inf _{\substack{a \neq b \\ a, b \in \mathbb{D}}}\left|\frac{1-\alpha^{2}}{(1-\alpha a)(1-\alpha b)}\right|=\frac{1-\alpha}{1+\alpha} .
$$

Now we consider the harmonic function $f(z)=\varphi(z)+\overline{g(z)}$, where $g(z)=\sum_{n=1}^{\infty} b_{n} z^{n}$ and the coefficients of $g$ satisfy the condition

$$
\sum_{n=1}^{\infty} n\left|b_{n}\right| \leq \frac{1-\alpha}{1+\alpha}
$$

We can easily see that (2.7) implies $f$ is sense-preserving in $\mathbb{D}$. For

$$
\left|g^{\prime}(z)\right|=\left|\sum_{n=1}^{\infty} n b_{n} z^{n-1}\right| \leq \sum_{n=1}^{\infty} n\left|b_{n}\right| \leq \frac{1-\alpha}{1+\alpha}<\frac{1-\alpha^{2}}{|1-\alpha z|^{2}}=\left|\varphi^{\prime}(z)\right| .
$$


By Corollary 2.5, it follows that $f$ is univalent in $\mathbb{D}$. We observe that $\varphi$ is a convex function and, by (2.7), $f$ is sense-preserving. Thus, by a result of Clunie and SheilSmall [2, Theorem 5.17], we conclude that the function $f$ in this case is close-toconvex in $\mathbb{D}$.

EXAMPLE 2.8. For $0<\alpha<1$, consider the harmonic function

$$
f_{a, \alpha}(z)=\frac{z-\alpha}{1-\alpha z}+\overline{a e^{i \beta} z+\left(\frac{1-\alpha}{1+\alpha}-a\right) e^{i \gamma} \frac{z^{2}}{2}}, \quad z \in \mathbb{D}
$$

where $\beta, \gamma$ are real, and $0<a<(1-\alpha) /(1+\alpha)$. As in Example 2.7, it can be easily seen that $f_{a, \alpha}(z)$ is sense-preserving in the unit disc $\mathbb{D}$ and a simple computation shows that (2.6) is satisfied. Thus, by Corollary $2.5, f_{a, \alpha}(z)$ is univalent and close-to-convex in $\mathbb{D}$.

\section{References}

[1] S. Axler, P. Bourdon and W. Ramey, Harmonic Function Theory, 2nd edn, Graduate Texts in Mathematics, 137 (Springer, New York, 2001).

[2] J. G. Clunie and T. Sheil-Small, 'Harmonic univalent functions', Ann. Acad. Sci. Fenn. Ser. A.I. 9 (1984), 3-25.

[3] P. L. Duren, Univalent Functions, Grundlehren der Mathematischen Wissenschaften, 259 (Springer, New York, 1983).

[4] P. Duren, Harmonic Mappings in the Plane, Cambridge Tracts in Mathematics, 156 (Cambridge University Press, Cambridge, 2004).

[5] H. Lewy, 'On the nonvanishing of the Jacobian in certain one-to-one mappings', Bull. Amer. Math. Soc. 42 (1936), 689-692.

[6] M. N. Pascu and N. R. Pascu, 'Neighbourhoods of univalent functions', Bull. Aust. Math. Soc. 83(2) (2011), 210-219.

[7] S. Ponnusamy, H. Yamamoto and H. Yanagihara, 'Variability regions for certain families of harmonic univalent mappings', Complex Var. Elliptic Equ. 58(1) (2013), 23-34.

VAIDHYANATHAN BHARANEDHAR, Department of Mathematics, Indian Institute of Technology Madras, Chennai-600 036, India

e-mail: bharanedhar3@gmail.com

SAMINATHAN PONNUSAMY, Department of Mathematics, Indian Institute of Technology Madras, Chennai-600 036, India

e-mail: samy@isichennai.res.in,samy@iitm.ac.in 